\title{
Level of Knowledge of Covid-19 among Dental Students of the Universidad Católica De Santiago De Guayaquil-Ecuador
}

\section{Margy A Guerra-Riofrio ${ }^{1 *}$, Fuad 0 Huamán-Garaicoa ${ }^{2}$ and Karla E Cruz-Moreira ${ }^{3}$}

${ }^{1}$ Dental School Graduate from Universidad Católica de Santiago de Guayaquil (UCSG), Guayaquil, Ecuador

${ }^{2}$ Pathologist and Professor of the School of Medicine at the Universidad Católica de Santiago de Guayaquil (UCSG), Guayaquil, Ecuador

${ }^{3}$ Specialized in Pediatrics and Oral Dentistry, Professor at the Oral Pathology Department of the Dental School at the Universidad Católica de Santiago de Guayaquil, Coordinator of the Community Affairs Department at the Universidad Católica de Santiago de Guayaquil (UCSG), Guayaquil, Ecuador

*Corresponding Author: Margy A Guerra-Riofrio, Dental School Graduate from Universidad Católica de Santiago de Guayaquil (UCSG), Guayaquil, Ecuador.
Received: March 15, 2021

Published: May 18, 2021

(C) All rights are reserved by Margy

A Guerra-Riofrio., et al.

\begin{abstract}
Objective: Determine the level of knowledge about COVID-19 among students from Dental School of the Universidad Católica de Santiago de Guayaquil (UCSG) in 2020.

Materials and Methods: A 14-item survey was given to 182 students from the Dental School UCSG corresponding to $6^{\text {th }}$ to $9^{\text {th }}$ semesters who volunteered to participate in the study. The questionnaire was divided in 6 sections about COVID-19 knowledge.

A score was assigned to measure the level of knowledge (equal or less than 3 points: bad, 4 - 6: medium, 7 - 8: good and 9 - 10: excellent). The total score was compared among the participants from different courses.

Data were analyzed in SPSS program $25^{\text {th }}$ version. To compare association between variables, ANOVA and correlation (Pearson's coefficient), T-Student and Chi-square tests were done.

Results: From the 182 volunteers, 81.86\% (149) obtained a score between 4 - 6.9 points, 14.83\% obtained a score between 7 - 8.99 points, $3.29 \%$ of the participants obtained equal or less than 3.99 points. The level of knowledge was not related to the academic period in which the students were enrolled.

Conclusion: This study reveals that Dentistry UCSG students have a medium level of basic knowledge about COVID-19. Knowledge was best among students in $8^{\text {th }}$ semester.

Keywords: Community Dentistry; COVID-19; Level of Knowledge, Attitudes, and Practice in Health; Coronavirus Infection; Dental Students
\end{abstract}

\section{Abbreviations}

UCSG: Universidad Católica de Santiago de Guayaquil; COE: National Emergency Operation Committee (COE, from spanish Comi- té Nacional de Operaciones de Emergencias); ACE-2: Angiotensin II Converting Enzyme Receptor; WHO: World Health Organization; COVID-19: Coronavirus Disease 2019; SARS-CoV-2: Severe Acute 
Respiratory Syndrome Coronavirus 2; PCR: Polymerase Chain Reaction; RT-PCR: PCR in Real Time

\section{Introduction}

On January seventh of 2020, the Chinese Centre for Disease Control and Prevention and the health authorities of Wuhan (China) identified a novel coronavirus; this resulted after a group of patients with an unknown pneumonia were seen in December and the collection of lower respiratory tract swabs revealed the genomic sequence of the virus, and they realized it was very similar to SARS-CoV from 2002. The International Committee on Taxonomy of Viruses named it as SARS-CoV-2; later, by February eleventh the World Health Organization (WHO) called its disease as COVID-19 [1].

The virus spread rapidly, on February twenty-sixth there were 80.239 cases and 2.700 deaths worldwide [2]. On March $30^{\text {th }}$, the WHO announced that the new outbreak had turned into a pandemic. In Ecuador, the first symptomatic case appeared on February $29^{\text {th }}$, meanwhile, on March $11^{\text {th }}$ the National Health Ministry declares a public health emergency since there were 447 confirmed cases on a national level [3]. The last report done by the National Emergency Operation Committee (COE, from spanish Comité Nacional de Operaciones de Emergencias) from August $21^{\text {st }}$ informed that there was a total of 106.481 confirmed cases with 6.248 deaths locally [3].

Out of all the health professionals, dentists have a higher transmission risk, because they are exposed on the daily practice to the main sources of transmission of the virus (air particles, aerosol) [4]; therefore, in Dentistry it is essential to have knowledge of how the virus is transmitted, its main symptoms, oral manifestations and established prevention biosafety protocols.

\section{Aim of the Study}

The aim of this study was to determine the level of knowledge about SARS-CoV-2 and COVID-19 among Dentistry students from an university of Guayaquil, which turns out of great significance because when the clinical practice return both students and teachers must be ready. This study is intended to highly contribute to dental school community, because there is no actual study related to this topic.

\section{Materials and Methods}

A cross-sectional study was conducted by using a 14-item survey which was previously validated by 10 health professionals.
A link: https://drive.google.com/file/d/12aMMotoZY8IVvfuiZz UgbwtZha8G6t44/view? usp=sharing was emailed to 228 students enrolled in Dental School, corresponding to $6^{\text {th }}$ to $9^{\text {th }}$ semesters (A2020 period) of the UCSG, to evaluate the level of knowledge of future dentists about COVID-19.

At the beginning of the survey an informed consent was accepted. The survey was sent in June and closed by the next month. In total, 182 students volunteered to take part in the study. Fourty-six students didn't returned the survey. All questionnaires received were fully accomplished. There was no missing data.

The fourteen questions was distributed in five sections. The first section, "Etiopathogenesis", included four questions (Q1-Q4) about etiologic agent, transmission and cell receptor. The second section, "Signs, symptoms and laboratory results", had three questions (Q5-Q7) about mild and severe systemic manifestations and oral manifestations. In the $3^{\text {rd }}$ section, "Diagnosis", three questions (Q8-Q10) related to sample site, rapid tests and specificity and sensitivity tests to virus' detection. The fourth section "Treatment" had two questions (Q11-Q12) about medications used to treat COVID-19 and their side effects in oral cavity, both questions without limits of answers. Finally, the fifth section corresponded to "Biosafety", two questions (Q13-Q14) about useful resources in main biosafety protocols (with maximum three answers) and their confidence about a possible return to clinical practices at the UCSG. The questions were multiple choice. Q2, Q4-Q6 and Q8-Q13 allow more than one answer.

To reach the goal to determine the level of knowledge of the participants a score was given to first, second and third sections of the survey. Each section was graded with 10 points resulting in a maximum of 30 points corresponding to a global score of 10/10. The treatment and biosafety sections were gone away the total score because there was no protocol accepted by the WHO at that time. The following scale was used: equal or less than 3: bad, 4 - 6: medium, 7 - 8: good and 9 - 10: excellent knowledge level. A total score equal or more than $7 / 10$ was considered approved (good and excellent).

We proposed the hypothesis: The $9^{\text {th }}$ academic term's students have more knowledge about COVID-19 than students from lower semesters.

Data from survey were exported to Excel. To compare association between variables, Pearson's correlation, Student's T and Chi- 
square tests were done. All statistical evaluations were performed using the software package SPSS (25 $5^{\text {th }}$ version). The analysis was done with a $95 \%$ confidence interval. For all tests, $\mathrm{p}<0.05$ was regarded as statistically significant.

The study was approved by the UCSG Academic and Ethics Committee and followed the ethical principles set forth in the 1964 Declaration of Helsinki for medical research on human beings. The confidentiality of the information recorded through the surveys was maintained and participant names were not identified at any time during the study. All individuals provided their informed written consent.

\section{Results}

The data set contained 182 volunteers, 122 (67\%) female and 60 (33\%) male. The median age was 24 (range: 20 - 35) years. Fifty-six $(31 \%)$ participants belong to $6^{\text {th }}$ semester, thirty-seven $(20 \%)$ to $7^{\text {th }}, 51(28 \%)$ to $8^{\text {th }}$ and $38(21 \%)$ to $9^{\text {th }}$ academic term.

In the first question about etiology agent for COVID-19, 77\% of the participants mentioned SARS-CoV-2. Concerning the route of transmission, participants could choose until three options. Ninety-two percent, $90,7 \%$ and $52,7 \%$ of the participants selected "air", "respiratory droplets" and "fomites", respectively. Regarding the question about human cell receptor for SARS-CoV-2, 52,2\% chose ACE-2 (angiotensin II converting enzyme receptor). In the last question of the Etiopathogenesis section about main tissue expression of ACE-2, they were asked to provide up to three answers, $81,9 \%$ selected "lungs", 43,4\% "major salivary glands" and 35,7\% "oral mucosa” (See table 1).

Graph 1 shows clinical manifestations and ancillary tests. About mild COVID-19 infections, 95.6\% chose "fever", 93.9\% "dry cough" and $81.8 \%$ "fatigue", meanwhile in severe infections participants mostly selected "bilateral lung opacities" (80.7\%), "lung infiltrates greater than $50 \%$ " (80.2\%), and "oxygen saturation equal to or less than $93 \%$ at rest" (49.4\%). Concerning oral signs and symptoms, majority $(73.6 \%)$ indicated there was no oral manifestations of COVID-19. Ten-percent volunteers indicated there was oral symptoms but didn't write any. The most prevalent oral manifestations reported were erosions $(4,4 \%)$, dysgeusia $(3,3 \%)$, and petechias $(2,2 \%)$.

In the Diagnosis section, about the best site of sample collection for COVID-19 virus' detection, 87.4\% said "nasopharynx", 87.4\% "blood", 50\% "saliva" and 37.4\% "oropharynx". Related to identify-

\begin{tabular}{|c|c|c|}
\hline & Question & Answers (Number; \%) \\
\hline Q1 & Etiology & $\begin{array}{l}\text { SARS-CoV-2 }(140 ; 77 \%) \\
\text { SARS-CoV-1 }(39 ; 21,4 \%) \\
\text { HCoV-HKU1 }(2 ; 1,1 \%) \\
\text { MERS-CoV }(1 ; 0,5 \%)\end{array}$ \\
\hline Q2 & $\begin{array}{l}\text { Transmission routes } \\
\qquad(\mathrm{n}=478)\end{array}$ & $\begin{array}{l}\text { Airway }(167 ; 91,8 \%) \\
\text { Respiratory droplets }(165 \text {; } \\
\qquad 90,7 \%) \\
\text { Fomites }(96 ; 52,7 \%) \\
\text { Oro-faecal }(33 ; 18,1 \%) \\
\quad \operatorname{Skin}(17 ; 9,3 \%)\end{array}$ \\
\hline Q3 & Cell human receptor & $\begin{array}{l}\text { ACE-2 }(95 ; 52,2 \%) \\
\text { ACE-1 }(55 ; 30,2 \%) \\
\text { DPP-4 }(32 ; 17,6 \%)\end{array}$ \\
\hline Q4 & $\begin{array}{c}\text { Main expression of ACE-2 } \\
\text { protein }(n=441)\end{array}$ & \begin{tabular}{|} 
Lungs $(149 ; 81,9 \%)$ \\
Major salivary glands $(79 ; 43,4 \%)$ \\
Oral mucosa $(65 ; 35,7 \%)$ \\
Oesophagus $(43 ; 23,6 \%)$ \\
Tongue $(43 ; 23,6 \%)$ \\
Jugal mucosa $(32 ; 17,6 \%)$ \\
Kidney $(17 ; 9,3 \%)$ \\
Liver $(8 ; 4,4 \%)$ \\
Teeth $(5 ; 2,7 \%)$
\end{tabular} \\
\hline
\end{tabular}

Table 1: Etiopathogenesis.

Symbology: Severe acute respiratory syndrome coronavirus 2 (SARS-CoV-2); Severe acute respiratory syndrome coronavirus 1 (SARS-CoV-1); Middle East Respiratory Syndrome CoV (MERS-CoV); Human coronavirus; Angiotensin converting enzyme 2 (ACE-2); Angiotensin converting enzyme 1 (ACE-1); dipeptidyl peptidase receptor-4 (DPP-4). Q2 and Q4 accepted more than one answer.

ing a rapid test to detect the virus, $45.6 \%$ said "blood antibodies", $30.2 \%$ "PCR" (polymerase chain reaction)" and 11.5\% "ELISA for 


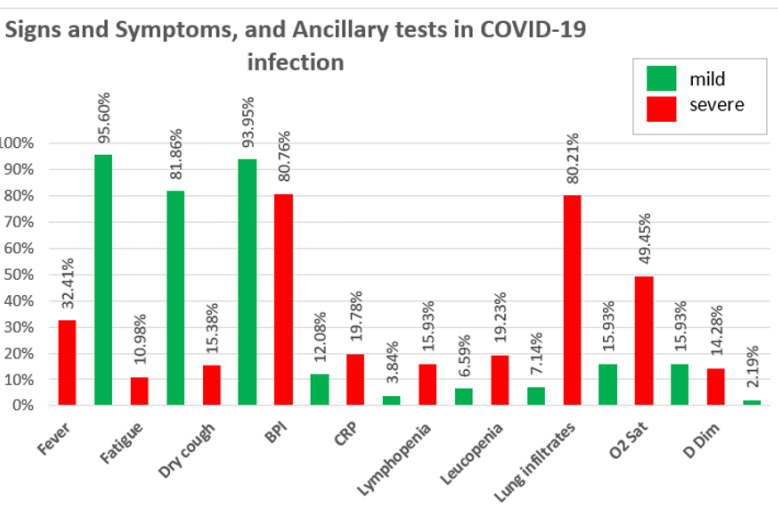

Graph 1: Mild and severe COVID-19 infection: Signs, symptoms and ancillary tests (Q5-Q6).

Symbology: Bilateral Pulmonary Infiltrates (BPI), elevated C-reactive protein (CRP), Lung Infiltrates $>50 \%$ (Lung infiltrates), Oxygen saturation $<93 \%$ at rest (O2 Sat), D Dimer (D Dim). Questions (Q5-Q6) accepted more than one answer.

COVID-19" (See graph 2). Fifty-two percent selected "PCR" as the most effective test, followed by $41 \%$ "blood antibodies" and $36 \%$ "ELISA".

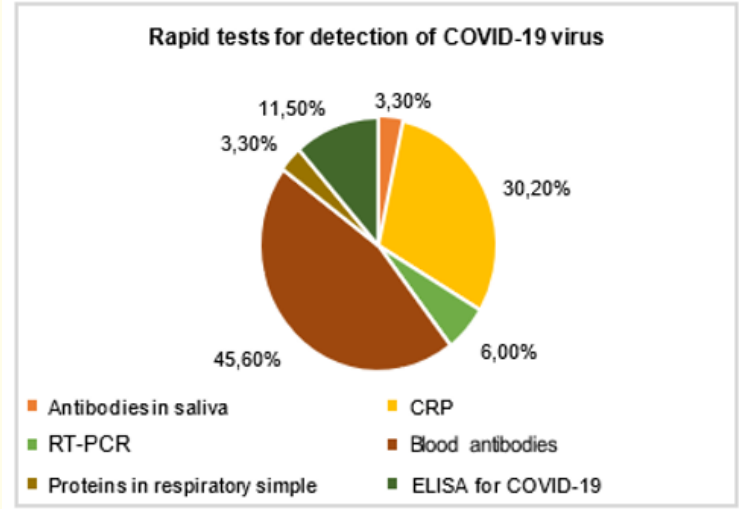

Graph 2: Lab tests for SARS-CoV-2 detection (Q9).

Symbology: Polymerase Chain Reaction (PCR), Real time- PCR (RT-PCR), Enzyme-link Immunoassay (ELISA).

Q9 accepted more than one answer.
At the Treatment section, participants were asked to select the drugs identified as being used to treat COVID-19. "Hydroxychloroquine" was the most selected (78.6\%), "chloroquine" by $43.4 \%$ and "remdesivir" by $26.9 \%$. About drug's side effects in the oral cavity, for hydroxychloroquine $36.5 \%$ chose "xerostomia" and $23.6 \%$ "none"; for remdesivir $12.1 \%$ "none" and $8.2 \%$ "xerostomia"; for chloroquine 23.1\% selected "xerostomia" and 9.9\% "none" (See table 2).

\begin{tabular}{|c|c|c|c|}
\hline \multirow[b]{2}{*}{ Q11 } & Question & \multicolumn{2}{|c|}{ Answers (Number; \%) } \\
\hline & $\begin{array}{l}\text { Drugs } \\
(n=271)\end{array}$ & $\begin{array}{c}\text { Hydroxychlo- } \\
\text { roquine (143; } \\
78,6 \%) \\
\text { Chloroquine (79; } \\
43,4 \%) \\
\text { Remdesivir (49; } \\
26,9 \%)\end{array}$ & \\
\hline & & $\begin{array}{l}\text { Hydroxychloro- } \\
\text { quine }(\mathrm{n}=170)\end{array}$ & $\begin{array}{l}\text { Xerostomia }(67 ; 36,5 \%) ; \\
\text { Hyperpigmentation (27; } \\
14,8 \%) ; \\
\text { Ulcers }(26 ; 14,3 \%) ; \\
\text { Pemphigus vulgaris (7; } \\
3,8 \%) ; \\
\text { None }(43 ; 23,6 \%)\end{array}$ \\
\hline Q12 & $\begin{array}{c}\begin{array}{c}\text { Oral side ef- } \\
\text { fects } \\
(n=319)\end{array}\end{array}$ & $\begin{array}{l}\text { Chloroquine } \\
\qquad(n=93)\end{array}$ & $\begin{array}{l}\text { Xerostomia }(42 ; 23,1 \%) ; \\
\text { Hyperpigmentation (16; } \\
8,8 \%) ; \\
\text { Ulcers }(13 ; 7,1 \%) ; \\
\text { Pemphigus vulgaris (4; } \\
2,2 \%) ; \\
\text { None }(18 ; 9,9 \%)\end{array}$ \\
\hline & & $\begin{array}{l}\text { Remdesivir } \\
\quad(n=56)\end{array}$ & $\begin{array}{c}\text { Xerostomia (15; 8,2\%) } \\
\text { Hyperpigmentation (8; } \\
4,4 \%) ; \\
\text { Ulcers (7; 3,8\%); } \\
\text { Pemphigus vulgaris (4; } \\
2,2 \%) ; \\
\text { None }(22 ; 12,1 \%)\end{array}$ \\
\hline
\end{tabular}

Table 2: Treatment.

Questions (Q11-Q12) accepted more than one answer. 
The Biosafety Protocols section was the last part of the survey. One of the questions consisted in choosing three products used in protocols for surface disinfection in a dental office. Fifty-eight percent selected the option "glutaraldehyde", 52.2\% "sodium hypochlorite" and 37.9\% "ethanol". Finally, they were asked if they felt safe to return to their pre-graduate practices, $86.3 \%$ answered "no" and $13.7 \%$ said "yes" (See table 3 ).

\begin{tabular}{|l|c|c|}
\hline & Question & Answers (Number; \%) \\
\hline Q13 & $\begin{array}{c}\text { Surface disinfection } \\
\text { product }\end{array}$ & $\begin{array}{c}\text { Glutaraldehyde }(106 ; 58,2 \%) \\
\text { Sodium hypochlorite }(95 ; 52,2 \%) \\
\text { Ethanol }(69 ; 37,9 \%) \\
\text { Ozone }(61 ; 33,5 \%)\end{array}$ \\
\hline $\begin{array}{c}\text { Q14 } \\
\text { Hydrogen peroxide }(53 ; 29,1 \%) \\
\text { return to pregraduate } \\
\text { practices }\end{array}$ & Chlorhexidine $(43 ; 23,6 \%)$ \\
\hline
\end{tabular}

Table 3: Biosafety protocols. Q13 accepted more than one answer.

To determine the level of knowledge of future dentists, those scored between 9 and 10 were considered to have an excellent knowledge about COVID-19 but no one obtained this score. Related to good knowledge level (8.99-7), 14.83\% obtained this score; meanwhile medium knowledge (6.99 - 4) was reached by the majority (81.86\%) and bad knowledge level (less than 3.99 ) by $3.29 \%$ of the participants.

A cross table was made considering the total score more than $7 / 10$ as a minimums score to qualifies with a knowledge medium/ high and the academic term of the student. Most of the students from $8^{\text {th }}$ semester obtained the highest score $40.7 \%$, followed by the students from $6^{\text {th }}(29.6 \%), 9^{\text {th }}(22.2 \%)$ and finally $7^{\text {th }}$ semester with $7.4 \%$ (chi square 0.2 ) (See table 4).

Once the assumptions of normality and homogeneity variances were verified, we conducted an ANOVA using the academic period in which the participants were as an independent variable and the average score of the knowledge of the participants in the sections of Etiopathogenesis, signs, symptoms, laboratory results and diagnosis as a dependent variable. The ANOVA revealed a $p$ value of

\begin{tabular}{|l|c|c|c|}
\hline Semester & $\begin{array}{c}\text { Approved ( } \mathbf{7}) \\
(\mathbf{N} ; \mathbf{\%})\end{array}$ & $\begin{array}{c}\text { Reproved (< 7) } \\
(\mathbf{N} ; \mathbf{\% )}\end{array}$ & Total (N; \%) \\
\hline VI & $8(29,6 \%)$ & $48(31 \%)$ & $56(30,77)$ \\
\hline VII & $2(7,4 \%)$ & $35(22,6 \%)$ & $37(20,32)$ \\
\hline VIII & $11(40,7 \%)$ & $40(25,8 \%)$ & $51(28,03)$ \\
\hline IX & $6(22,2 \%)$ & $32(20,6 \%)$ & $38(20,88)$ \\
\hline Total & $27(100)$ & $155(100)$ & $182(100)$ \\
\hline
\end{tabular}

Table 4: Total score according to academic term/semester.

0.61 , therefore the null hypothesis was not rejected; thus, the academic term in which the student is (sixth, seventh, eighth or ninth semester) does not influence the obtained score about COVID-19 knowledge.

\section{Discussion}

This study revealed that the general knowledge about COVID-19 among students from sixth to ninth academic term of Dental School is below expected. However, the results obtained were optimistic in some sections of the survey, such as the identification of the etiologic pathogen of the disease, because most of the participants selected SARS-CoV-2, name assigned by the International Commission of Viral Taxonomy [1], this matches with Brito., et al. study carried out in Brazil where approximately above half of the population selected the same answer [5].

In the second question about the main transmission pathways, the knowledge was poor, contrasting with the study of Karaaslan., et al. where students from Turkia perfectly understood the transmission mechanisms [6]. In our study most of the participants selected "airway" as transmission, this could be due to lack of knowledge about the definition of this term, according to the WHO, because in the context of COVID-19, airway transmission could be possible under specific circumstances and environments, where procedures or support treatments are conducted which can generate aerosols [7]; therefore, this pathway is not considered the main transmission. Many authors agree that the main transmission is by respiratory droplets, orofecal, fomites, etc [7-9].

In the etiopathogenesis section, related to cellular human receptor of the virus, only half of the participants selected ACE-2. Approximately, $75 \%$ of the participants identified the "lungs" as the main receptor expressing tissue, which is correct [10], however, regarding the main receptor expressing tissue in the oral cavity, the 
most selected answer was salivary glands and oral mucosa, thus revealing unawareness from the dental students because most of the studies suggest that the expression of ACE- 2 in the oral cavity is specifically in the tongue [10]. We believe that most of our participants selected salivary glands because they associated the saliva as a transmission way, thus creating some confusion, however, there is expression of ACE-2 receptors in the salivary glands and oral mucosa, but these are more prevalent in the tongue [10].

Upon analysis of the signs, symptoms, and laboratory results section, we noticed that knowledge of the mild and severe clinical manifestations of COVID-19 was optimal except that most of the participants also included fever as an alarming sign, which by some authors is classified among the mild manifestations. Regarding the ancillary tests that they would request for patients with mild and severe manifestations we found unawareness in the initial stages of the disease. The students do not consider requesting a complete blood count and acute phase reactants test as a first option upon fever, dry cough and fatigue, which is important because according to authors like Tang., et al. leukopenia, lymphopenia and elevated C-reactive protein are present in the early stages of COVID-19 [9] and soliciting these tests will facilitate a rapid intervention to establish an early diagnosis. On the other hand, it should be noted that all the participants were aware of the tests needed to be requested in the late stages of the disease, these results were similar to those reported by Wu., et al. and Berlin., et al. who indicate that the tests indicating severity are blood oxygen saturation equal or less than 93\%, lung infiltrates greater than 50\% [11,12], bilateral lung opacities found in radiographic studies, elevated C-reactive protein, and elevated D dimer $[13,14]$.

The majority of our volunteers indicated there is no oral manifestations in COVID-19. Should be noted that this topic has not been asked in similar studies currently, just as other topics such as: rapid and specific tests, side effects of medications in oral cavity and surface disinfecting substances used in a dental office. The reason for this answer could be because at the time the survey was not enough evidence reported in the literature about this. However, studies like Sousa., et al. indicate the relevance to increase more studies with a bigger population groups because there is not estimation of the average of patients with COVID-19 presenting oral lesions [15]. In other hand, many authors have not been able to determine yet if these manifestations are associated with the virus or are secondary lesions to the treatment [16-18].
In Diagnosis section, our participants clearly recognized the sites of sample collection to detect the virus; however, most did not recognize which were the rapid tests to detect SARS-CoV-2. Most of the subjects $54.4 \%$ selected tests as antibodies in saliva, PCR and ELISA for COVID-19 and only 45.6\% indicated blood antibodies which is one of the most used rapid tests. This reveals the lack of information differentiating between a rapid test and a greater complexity test, since PCR and ELISA for COVID-19 are not considered as rapid tests $[19,20]$. Regarding the higher sensitivity and specificity tests, only a quarter of the population identified RT-PCR (PCR in real time) and ELISA for COVID-19 as higher confidence tests, this could be due to the lack of familiarity with the names of the tests.

In the treatment section, our objective was to ask about the knowledge regarding medications. In the question: which are the medications used to treat the disease? More than $75 \%$ of the participants indicated Hydroxychloroquine and Chloroquine; but when we asked about the side effects of these in the oral cavity a great unawareness was noted, the possible explanation for these results could be because these medications are not commonly used in the dental practice. However, it is relevant to know the side effects of different medications in the oral cavity, even more when there is sufficient scientific evidence about the antimalarial drug lesions $[16,21,22]$, as seen in the study from Bahloul., et al. where they found skin hyperpigmentation in $65.5 \%$ and in mucosae in $12 \%$ [22], on the other hand, in the study of Jallouli., et al. pigmented lesions were reported in $92 \%$ of their series [23].

In the last section, biosafety, it was found that the opinions regarding disinfecting substances were varied, being glutaraldehyde the product most chosen to be used in the dental office. It must be noted that Kampf., et al. and others authors agreed that the first line substances to significantly reduce coronavirus in surfaces are sodium hypochlorite at $0,1 \%$ and ethanol at $62-71 \%$ in an exposure range of 1 minute. However, the same author did not reject the effectivity of other substances [24].

The other question from this section was made with the objective of knowing if the participants felt safe to return to their pre-graduate practice, with most of them answering negatively, which supports the Brito., et al. study. conducted in Brazil where the participants from Dentistry also indicated feeling "worried about going back" [5]. This could be due to the great fear that hands on activities imply, because the risk is very high among dentists and Dental School students who are in close contact with the oral 
cavity and manipulate instruments that generate great amount of bioaerosols [2]. In addition, South American countries such as Brazil and Ecuador were severely affected, and therefore the fear among their habitants is evident and is greater in the most affected cities, such as Guayaquil in Ecuador [5].

Finally, we could determine that the level of knowledge about COVID-19 in the Etiopathogenesis, signs, symptoms, lab results, and diagnosis among the participants of the dental school is medium score. In this study the participants had more difficult in response the second and thirds section of the survey. These results were similar to those reported by Kashid., et al. where students from Dental college of India also had a poor knowledge about transmission mechanisms and diagnosis [25]; but the results obtained from this study are not in accordance with other studies like Chandra., et al. from the University at North India, because in his study $85,8 \%$ subjects obtained a good score of knowledge in this topics $[5,6,26]$. Therefore, the level of knowledge would not be related to a geographical area, because in the same country we can find that students from an institution dominate the topic about COVID-19 better than others. Rather, the interest in a subject related to COVID-19 could be the answer of why that some sections of the survey were answered in a better way.

On the other hand, we found that there is no significant difference regarding the total score of knowledge about COVID-19 and the academic term in which the student is enrolled, these is similar to those reported by Chandra [26], but differing with Jha., et al. where the scores from the medical and dental students in Lalitpur, Nepal from higher terms were significantly better than the ones in lower terms [27].

\section{Conclusion}

This work had made possible to know that students from the Dental School at the UCSG have a medium level of knowledge about COVID-19 infection, thus, motivation is important to persuade them to investigate everything concerning this virus, specially about the biosafety measures that must be taken in the dental practice. It is also important that radio, television, webpages and social networks make sure to publish the correct information regarding the various topics of COVID-19 infection since in this way, not only students but the population in general can know about this current problem.

\section{Bibliography}

1. Park S. "Epidemiology, virology, and clinical features of severe acute respiratory syndrome-coronavirus-2 (SARS-CoV-2;
Coronavirus Disease-19)". Pediatric Infection and Vaccine 27.1 (2020): 1-10.

2. Meng L., et al. "Coronavirus Disease 2019 (COVID-19): Emerging and Future Challenges for Dental and Oral Medicine". Journal of Dental Research 99.5 (2020): 481-487.

3. El MSP informa: Situación coronavirus COVID-19 (21-082020) - Ministerio de Salud Pública (2020).

4. Sabino-Silva R., et al. "Coronavirus COVID-19 impacts to dentistry and potential salivary diagnosis". Clinical Oral Investigations 24.4 (2020): 1619-1621.

5. Brito M., et al. "Brazilian dental students and COVID-19: a survey on knowledge and perceptions". Dentistry and Oral Medicine (2020).

6. Karaaslan F., et al. "Comparison of COVID-19 Relevant Knowledge and Attitudes of Clinical and Preclinical Dental Students in Turkey". Balkan Journal of Dental Medicine (2020): 24.

7. WHO. "Modes of transmission of virus causing COVID-19: implications for IPC precaution recommendations" (2020).

8. Helmy Y., et al. "The COVID-19 Pandemic: A Comprehensive Review of Taxonomy, Genetics, Epidemiology, Diagnosis, Treatment, and Control". Journal of Clinical Medicine 9.4 (2020).

9. Tang D., et al. "The hallmarks of COVID-19 disease". PLOS Pathogens 16.5 (2020).

10. Xu H., et al. "High expression of ACE2 receptor of 2019-nCoV on the epithelial cells of oral mucosa". International Journal of Oral Science 12.1 (2020): 8.

11. Wu Z and McGoogan JM. "Characteristics of and Important Lessons From the Coronavirus Disease 2019 (COVID-19) Outbreak in China: Summary of a Report of 72314 Cases From the Chinese Center for Disease Control and Prevention". The Journal of the American Medical Association 323.13 (2020): 1239-1242.

12. Berlin D., et al. "Severe Covid-19". The New England Journal of Medicine (2020).

13. Chen G., et al. "Clinical and immunological features of severe and moderate coronavirus disease 2019". Journal of Clinical Investigation 130.5 (2020): 2620-2629. 
14. Rodriguez A., et al. "Clinical, laboratory and imaging features of COVID-19: A systematic review and meta-analysis". Travel Medicine and Infectious Disease 34 (2020): 101623.

15. Sousa F and Paradella T. "Considerations on oral manifestations of COVID-19”. Journal of Medical Virology (2020).

16. Diaz M., et al. "Oral manifestations associated with COVID-19". Oral Diseases (2020): 1-3.

17. Amorim Dos Santos J., et al. "Oral mucosal lesions in a COVID-19 patient: New signs or secondary manifestations?" International Journal of Infectious Diseases 97 (2020): 326-328.

18. Martín C., et al. "Oral vesiculobullous lesions associated with SARS-CoV-2 infection". Oral Diseases (2020).

19. Udugama B., et al. "Diagnosing COVID-19: The Disease and Tools for Detection”. ACS Nano (2020).

20. Mak GC., et al. "Evaluation of rapid antigen test for detection of SARS-CoV-2 virus". Journal of Clinical Virology 129 (2020): 104500.

21. Chacón V., et al. "Oral manifestations associated with antimalarial therapy in patients with systemic lupus erythematosus". Lupus 29.7 (2020): 761-766.

22. Bahloul E., et al. "Hydroxychloroquine-induced hyperpigmentation in systemic diseases: prevalence, clinical features and risk factors: a cross-sectional study of 41 cases". Lupus 26.12 (2017): 1304-1308.

23. Jallouli M., et al. "Hydroxychloroquine-Induced Pigmentation in Patients With Systemic Lupus Erythematosus: A Case-Control Study". JAMA Dermatology 149.8 (2013): 935-940.

24. Kampf G., et al. "Persistence of coronaviruses on inanimate surfaces and their inactivation with biocidal agents". Journal of Hospital Infection 104.3 (2020): 246-251.

25. Kashid R., et al. "Awareness of COVID-19 amongst undergraduate dental students in India -A questionnaire based cross-sectional study". Research Square (2020).

26. Chandra DJ. "Knowledge, Attitude, And Behavior Of Covid-19 Among Dental Students At North India Dental College- A Questionnaire Survey". Ejbps 7.5 (2020): 558-561.
27. Jha N., et al. "Knowledge about the COVID-19 pandemic among undergraduate medical and dental students in Lalitpur, Nepal". Journal of Chitwan Medical College 10.33 (2020): 69-73.

\section{Volume 5 Issue 6 June 2021}

(c) All rights are reserved by Margy A Guerra-Riofrio., et al. 